\title{
ALGEBRAICALLY CLOSED GROUPS
}

\author{
W. R. SCOTT
}

1. Introduction. Let $G$ be a group with identity $e$. If $g \in G$, $g \neq e$, then there is no solution of the equation $x^{-1} x=g$ in any group $H$ containing $G$ as a subgroup. Thus the amount of algebraic closure which a group can have is somewhat limited. One of the possible definitions of algebraically closed groups is considered in $\$ 2$. The principal result is that every group $G$ is a subgroup of an algebraically closed group $H$. Generalizations are given in \$3. Another possible definition of algebraically closed groups is considered briefly in $\$ 4$.

Neumann $[1]^{1}$ has considered the related problem of adjoining roots of equations to groups. He gave necessary and sufficient conditions for the possibility of such adjunctions. Only incidental use will be made of his results in this paper, however.

2. Algebraically closed groups. Let $G$ be a group with identity $e$, and let $W\left(x_{j}, g_{k}\right)$ be a word in the indeterminates $x_{j}(j=1, \cdots, s)$ and elements $g_{k} \in G(k=1, \cdots, t)$.

Definition. A group $G$ is algebraically closed (briefly a.c.) if and only if for every finite set

$$
\begin{array}{lr}
W_{i}\left(x_{j}, g_{k}\right)=e & (i=1, \cdots, r), \\
W_{i}\left(x_{j}, g_{k}\right) \neq e & \left(i=r+1, \cdots, r^{\prime}\right)
\end{array}
$$

of equations and inequalities it is true that either

(i) There exist $x_{j} \in G(j=1, \cdots, s)$ satisfying (1), or

(ii) If $H$ is a group containing $G$ as a subgroup, then there do not exist $x_{j} \in H(j=1, \cdots, s)$ satisfying (1).

Clearly no group of finite order is a.c. In the following, the order of a group will mean the (cardinal) number of elements in the group.

Lemma 1. If $G$ is a group, then there exists a group $H$ such that the following conditions are satisfied.

(a) $G$ is a subgroup of $H$.

(b) If (1) is any set of equations and inequalities with coefficients in $G$ then either

(i) There is a solution of (1) in $H$, or

(ii) There is no solution of (1) in any group $K \supset H$.

Received by the editors February 7, 1949 and, in revised form, January 13, 1950 .

${ }^{1}$ Numbers in brackets refer to the bibliography at the end of the paper. 
(c) The order of $H$ is the larger of $\aleph_{0}$ and the order of $G$.

Proof. It may be assumed that $G$ is infinite. Let $A$ be the order of $G$. Then there are just $A$ distinct systems (1). Well order them: $\left\{(1)_{\alpha}\right\}(\alpha=1,2, \cdots)$. Let $G_{0}=G$. Suppose inductively that $G_{\gamma}$ has been defined for $\gamma<\alpha$ so that $G_{\gamma}$ is of order $A$ for all such $\gamma$, and so that $G_{\beta} \subset G_{\gamma}$ for $\beta<\gamma$. Let $K_{\alpha}=\cup G_{\gamma}, \gamma<\alpha$. Then $K_{\alpha}$ is of order $A$. If (1) $)_{\alpha}$ has no solution in any group $K \supset K_{\alpha}$, then let $G_{\alpha}=K_{\alpha}$. If (1) $)_{\alpha}$ has a solution $k_{1}, \cdots, k_{s}$ in some group $K \supset K_{\alpha}$, let $G_{\alpha}$ be the subgroup of $K$ generated by $K_{\alpha}$ and $k_{1}, \cdots, k_{s}$. Thus in any case $G_{\alpha}$ has been defined inductively so that $G_{\alpha}$ is of order $A$ and so that $G_{\beta} \subset G_{\alpha}$ for $\beta<\alpha$. Set $H=\cup G_{\alpha}$. Then $H$ satisfies conditions (a), (b), and (c).

THEOREм 1. If $G$ is a group, then there exists a group $G^{*}$ such that the following conditions are satisfied.

(a) $G$ is a subgroup of $G^{*}$.

(b) $G^{*}$ is algebraically closed.

(c) The order of $G^{*}$ is the larger of $\aleph_{0}$ and the order of $G$.

Proof. Let $G=G^{(0)}$ and let $G^{(1)}$ be the group $H$ whose properties are listed in Lemma 1. In general, if $G^{(m)}$ has been defined, let $G^{(m+1)}$ be the group whose existence is guaranteed by Lemma $1, G^{(m)}$ being substituted for $G$ in that lemma. Let $G^{*}=\bigcup G^{(m)}$. Then conditions (a) and (c) are clearly satisfied. Any set (1) of equations and inequalities with coefficients in $G$ has all of its coefficients in some $G^{(m)}$. Therefore, by Lemma 1 , (1) either has a solution in $G^{(m+1)}$ and hence in $G^{*}$, or has no solution in any group $K \supset G^{(m+1)}$ and hence no solution in any group $K \supset G^{*}$. Thus $G^{*}$ is a.c.

THeOREM 2. If $G$ is an algebraically closed group and $H$ is a finite group, then there is a subgroup of $G$ isomorphic to $H$.

Proof. Let the elements of $H$ be $h_{1}, \cdots, h_{n}$, and let $W_{i}\left(h_{j}\right)$ $\left(i=1, \cdots, n^{2}\right)$ be the $n^{2}$ words of the form $h_{j_{1}} h_{j_{2}} h_{j_{3}}{ }^{-1}$ where $h_{j_{3}}=h_{j_{1}} h_{j_{2}}$. Consider the system

$$
\begin{array}{rr}
W_{i}\left(x_{j}\right)=e & \left(i=1, \cdots, n^{2} ; j=1, \cdots, n\right), \\
x_{j_{1}} x_{j_{2}}^{-1} \neq e & \left(j_{1} \neq j_{2} ; j_{1}, j_{2}=1, \cdots, n\right) .
\end{array}
$$

There is a solution of (2) in the direct product $G \times H$. Considering $G$ as a subgroup of $G \times H$, it follows that there is a solution $g_{1}, \cdots, g_{n}$ of (2) in $G$ since $G$ is a.c. But then the subgroup of $G$ with elements $g_{1}, \cdots, g_{n}$ is isomorphic to $H$.

3. Generalizations. In the definition of a.c. groups, the restriction 
to a finite set of equations and inequalities can be eased somewhat. Thus let $A$ be any infinite cardinal, and allow any set of fewer than $A$ equations and inequalities in (1). The concept thus defined will be called an a.c. (A) group. Thus an a.c. group is an a.c. $\left(\boldsymbol{N}_{0}\right)$ group and conversely. An a.c. (A) group is an a.c. (B) group for $B<A$.

Analogues of Theorems 1 and 2 are true, but no strong statement can be made as to the order of $G^{*}$ in Theorem 1 (at least not with the argument used here).

THEOREM 3. If $G$ is a group, then there exists an a.c. (A) group $G^{*}$ containing $G$ as a subgroup.

The proof is similar to that of Theorem 1. The analogue of Lemma 1 (with conclusion (c) omitted) offers no difficulty. In the proof of the main theorem, the simply infinite sequence $\left\{G^{(m)}\right\}$ is replaced by a well ordered sequence $\left\{G^{(\alpha)}\right\}$ with $B$ terms, where $B$ is the smallest cardinal greater than $A$.

TheOREM 4. If $G$ is an a.c. (A) group and $H$ is a group of order $B<A$, then there is a subgroup of $G$ isomorphic to $H$.

This theorem is a direct generalization of Theorem 2, and its proof is strictly analogous to that of Theorem 2 .

4. Weak closure. Call a group $G$ weakly algebraically closed (w.a.c.) if it satisfies the definition of algebraic closure with only equations permitted in (1). Then an a.c. group is w.a.c. The group of order 1 is w.a.c. No other group of finite order is w.a.c. For let $G$ be of order $n>1$ and let $g \neq e$ be an element of $G$. Then the equation

$$
x^{n} g^{-1}=e
$$

has no solution in $G$. On the other hand (see [1, Theorem 5.1]) there exists a group $H \supset G$ in which (3) has a solution. Hence $G$ is not w.a.c.

There remains the question as to whether there are other w.a.c. groups which are not a.c., and if so whether Theorem 2 holds in any form for w.a.c. groups.

5. Additional remarks. The referee has pointed out the facts given in this section. Let $G^{\prime}$ be the subgroup of $G$ generated by the elements $g_{k}$ appearing in (1). Then an equivalent definition of a.c. groups can be made by replacing the alternative (ii) in the previous definition by

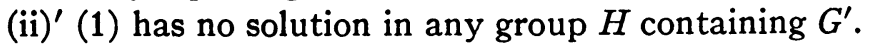

It is clear that if $G$ is a.c. in the second sense, it is a.c. in the first sense. Conversely, let $G$ be a.c. in the first sense. Suppose that (1) 
has no solutions in $G$. Let $H$ be a group containing $G^{\prime}$. Let $F$ be the free product of $G$ and $H$ with amalgamated subgroup $G^{\prime}$ (see [2, pp. 164-168]). Then, since $G$ is a subgroup of $F$, there is no solution of (1) in $F$, and hence none in $H$. Therefore $G$ is a.c. in the second sense, that is, the two definitions are equivalent.

Theorem 2 is valid for all groups $H$ which are definable by a finite number of generators, relations, and inequalities. I am unable to determine whether such a group can be infinite.

If "group" is replaced by "operator group" in the definition of algebraic closure and in Theorem 1, then Theorem 1 remains true if conclusion (c) is omitted.

Finally, it should be noted that most of the discussion applies to a large class of abstract algebras.

\section{BIBLIOGRAPHY}

1. B. H. Neumann, Adjunction of elements to groups, J. London Math. Soc. vol. 18 (1943) pp. 4-11.

2. O. Schreier, Die Untergruppen der freien Gruppen, Abh. Math. Sem. Hamburgischen Univ. vol. 5 (1927) pp. 161-183.

UNIVERSITY OF MichigAN 